\title{
REVIEW ARTICLE OPEN The regulation of protein translation and its implications for cancer
}

\author{
Ping Song ${ }^{1}$, Fan Yang ${ }^{2}$, Hongchuan $\operatorname{Jin}^{3}$ and Xian Wang ${ }^{1}$
}

In addition to the deregulation of gene transcriptions and post-translational protein modifications, the aberrant translation from mRNAs to proteins plays an important role in the pathogenesis of various cancers. Targeting mRNA translation are expected to become potential approaches for anticancer treatments. Protein translation is affected by many factors including translation initiation factors and RNA-binding proteins. Recently, modifications of mRNAs mainly N6-methyladenine $\left(\mathrm{m}^{6} \mathrm{~A}\right)$ modification and noncoding RNAs, such as microRNAs and long noncoding RNAs are involved. In this review, we generally summarized the recent advances on the regulation of protein translation by the interplay between mRNA modifications and ncRNAs. By doing so, we hope this review could offer some hints for the development of novel approaches in precision therapy of human cancers.

Signal Transduction and Targeted Therapy (2021)6:68

; https://doi.org/10.1038/s41392-020-00444-9

\section{INTRODUCTION}

Cancer is a major global public health problem and the second leading cause of death worldwide. ${ }^{1}$ It has been recognized as a disease resulting from the accumulation for multiple genetic and epigenetic changes. Genetic mutations mainly alter the functions of corresponding proteins, while epigenetic changes will change the expression of potential oncogenes and tumor suppressor genes. The regulation and function of these alterations have been substantially explored. ${ }^{2-7}$ However, the relevance of protein translation or the production of nascent proteins from mRNAs to the initiation and progression of human cancers used to be largely overlooked. With the recent development of experimental technologies, the regulation of protein translation has been gradually revealed. It could be affected by various factors including well-recognized players such as translation initiation factors and RNA-binding proteins, as well as new players such as noncoding RNAs and m6A modification of mRNAs (Fig. 1). In this review, we aimed to summarize the new advances in the regulation of protein translation, especially the contribution of dysregulated ncRNAs to the aberrant protein translation in cancers and facilitate a better understanding of the mechanism of carcinogenesis.

\section{OVERVIEW OF THE PROTEIN TRANSLATION PROCESS}

Translation steps

In general, protein translation can be divided into three main steps: initiation, extension, and termination. Initiation is the ratelimiting step in translation, and the formation of the translation initiation complex (elF4F) is the most critical process. ${ }^{8}$ elF4F consists of elF4A, elF4E, and elF4G. Among them, elF4A is a helicase that expands RNA into a single strand through the RNA- binding protein elF4B. elF4E is a cap-binding protein and elF4G is a scaffolding protein used for mechanical assembly. elF4F assembles on the structure of $5^{\prime}$ m7G-capped of RNA and interacts with polyA tail-binding protein (PABP). However, the capindependent mRNA translation process, which is IRES (internal ribosome entry site)-dependent translation, is triggered when the mTOR signaling pathway is inhibited by rapamycin, nutritional deficiencies, or hypoxia. As a result, activated 4EBP1 (dephosphorylation) binds to elF4E and inhibits cap-dependent translation. Meanwhile, elF4G expression increases and mediates IRESdependent translation. After the translation initiation complex is assembled, the $40 \mathrm{~S}$ ribosomal subunit binds to other initiation factors, including elF5 and the ternary complex (eIF2-GTP-MettRNAi) to form a $43 \mathrm{~S}$ ribosome, thus enabling the progression into the extension stage. ${ }^{9,10}$ The initiation complex of cap-dependent translation scans the mRNA from the 5 'end to find the first start codon (AUG), and enters the ribosomal P-site via initiator methionyl tRNA (Met-tRNAi). After that, the $43 \mathrm{~S}$ ribosome was recruited to the $5^{\prime}$ cap structure of $m R N A$ to form a $48 S$ ribosomal complex via elF3 complex interacting with elF4G. Meanwhile, GTP bound by elF2 (composed of $\alpha-, \beta-$, and $\gamma$ subunits) is activated by hydrolysis, leading to the release of elF2-GDP after 605 ribosomes are recruited, leaving the active $80 \mathrm{~S}$ ribosomes $(40 \mathrm{~S}$ and $60 \mathrm{~S}$ subunits) to enter the extension phase. ${ }^{11,12}$

Termination: when the mRNA with stop codon (UAG, UGA, UAA) enters the ribosomal A-site, it can't be recognized by activated amino acid (aa-tRNA). Instead, the protein releasing factor binds to the A-site, leading to the termination of protein synthesis ${ }^{13}$ (Fig. 2).

\section{Translation components}

In addition to mRNA as a template for protein translation, ribosomal rRNA is required to provide translation sites, and

\footnotetext{
'Department of Medical Oncology, Cancer Institute of Zhejiang University, Sir Run Run Shaw Hospital, Zhejiang University School of Medicine, Hangzhou, Zhejiang, China; ${ }^{2}$ Department of Hepatobiliary and Pancreatic Surgery, First Affiliated Hospital, Zhejiang University School of Medicine, Hangzhou, Zhejiang, China and ${ }^{3}$ Key Lab of Biotherapy in Zhejiang, Sir Run Run Shaw Hospital, Zhejiang University School of Medicine, Hangzhou, Zhejiang, China

Correspondence: Hongchuan Jin (jinhc@zju.edu.cn) or Xian Wang (wangx118@zju.edu.cn)

These authors contributed equally: Ping Song, Fan Yang.
}

Received: 27 June 2020 Revised: 30 August 2020 Accepted: 6 December 2020

Published online: 18 February 2021 
transfer RNA (tRNA) as a tool for amino acid transport. ${ }^{14,15}$ tRNA carries specific amino acids to the ribosome under the action of aminoacyl tRNA synthetase, and accurately recognizes the codon sequence on mRNA by its anticodon, and adds specific amino acids into the peptide. ${ }^{16}$ Aberrant expression of rRNA and tRNA during tumorigenesis is frequently found in different tumors, including colorectal cancer, ${ }^{17}$ rhabdomyosarcoma, ${ }^{18}$ and retinoblastoma. ${ }^{19}$ There are only 61 types of tRNAs involved in the process of protein translation in eukaryotic cells. Due to the complexity of the secondary structures of tRNAs, it is difficult to design the primer and the research on tRNAs has been greatly restricted. However, with the development of high-efficiency

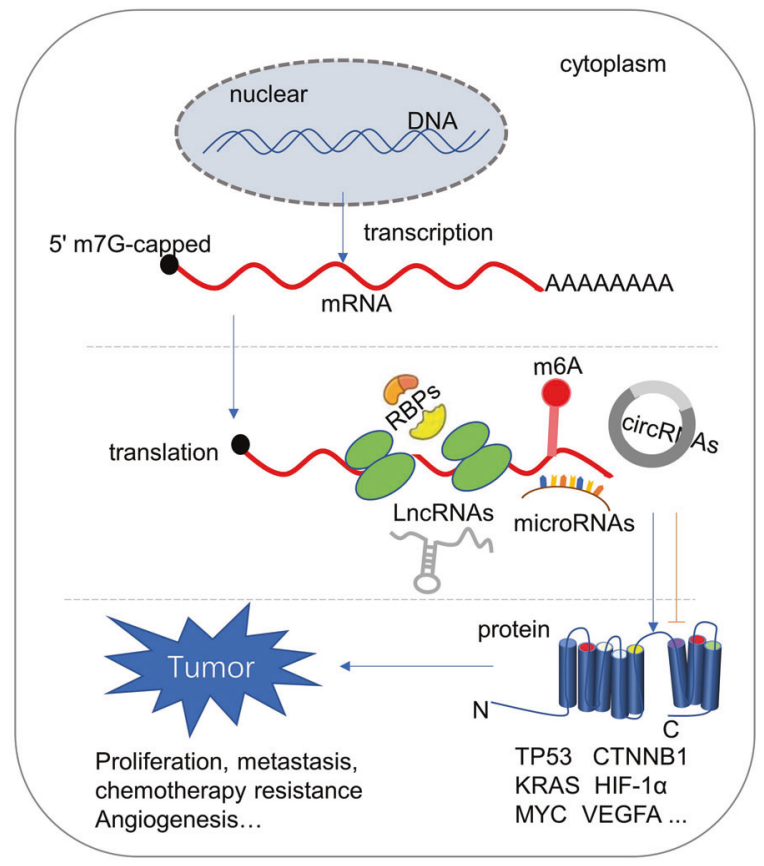

Fig. 1 The process of gene expression. Gene expression needs to be transcribed from DNA to RNA, translated into protein, and modified into mature protein to perform biological functions. This figure highlights the influence of RBPs, microRNAs, IncRNAs, circRNAs, and RNA m6A modification on the protein translation procedure in tumorigenesis reverse transcriptase enzymes, ${ }^{20}$ the exploration of tRNAs such as tRNA sequencing and quantification gradually became possible and popular.

The role of translation initiation factors in carcinogenesis Increased abundance of components of translation initiation complex is prevalent in various kinds of tumors. ${ }^{21}$ For example, in BRAF-mutated tumors, increased expression of elF4F complex leads

to anti-BRAF therapy resistance and more metastasis. Blocking the elF4E-elF4G interaction or targeted-inhibition of elF4A synergized BRAF-targeting therapy. Therefore, elF4F can be used as an indicator of acquired resistance to BRAF (V600) targeting therapy. ${ }^{22}$

The binding of elF4E to the $5^{\prime} \mathrm{m} 7 \mathrm{G}$-capped of the mRNA is the rate-limiting step for translation initiation. The genome-wide translation profiling reveals that the content of elF4E is crtical for the translation of mRNAs that regulate the reactive oxygen species (ROS), which promotes cancer cell survival. ${ }^{23}$ By degrading the mRNA of elF4E-BP2, which negatively regulates elF4E, IGF2BP3 promotes elF4E-mediated translation activation, and ultimately leading to tumor cell proliferation. ${ }^{24}$ Phosphorylated elF4E promotes protein translation and also plays an important role in antitumor immunotherapy. In a liver cancer model induced by MYC overexpression combined with KRAS mutation (G12D), cancer cells escape immune surveillance and suppress the cytotoxicity of CD8 + T cells by increasing PD-L1 protein translation. While, eFT508, in phase 2 clinical trials (NCT02937675, NCT02605083 and NCT03258398), inhibits elF4E phosphorylation to reduce the translation of PD-L1 protein, reverses the aggressive and metastatic characteristics. ${ }^{25}$ In summary, activated elF4E promotes the RNA translation to accelerate the development of cancer by enhancing proliferation, inducing resistance to chemotherapy and anti-immune therapy. Encouragingly, there are small-molecule inhibitors available to target elF4E in the clinical trials, such as elF4E antisense oligonucleotides (ASOs), in phase 2 clinical trials (NCT02605083), targeted the elF4E mRNA for destruction, proven to inhibit tumor growth. ${ }^{26,27}$

The hypophosphorylated form of elF4E-binding proteins (4EBPs) competes with elF4G to bind elF4E, which affects the formation of elF4F complex and inhibits translation. 4E-BPs inhibit the translation of proliferation and cell cycle proteins to affect tumor cell proliferation and growth. ${ }^{28,29}$ As a small-molecule inhibitor of the elF4E/elF4G interaction, 4EGI-1 can mimic 4E-BP function to restrain cap-dependent translation and inhibit the

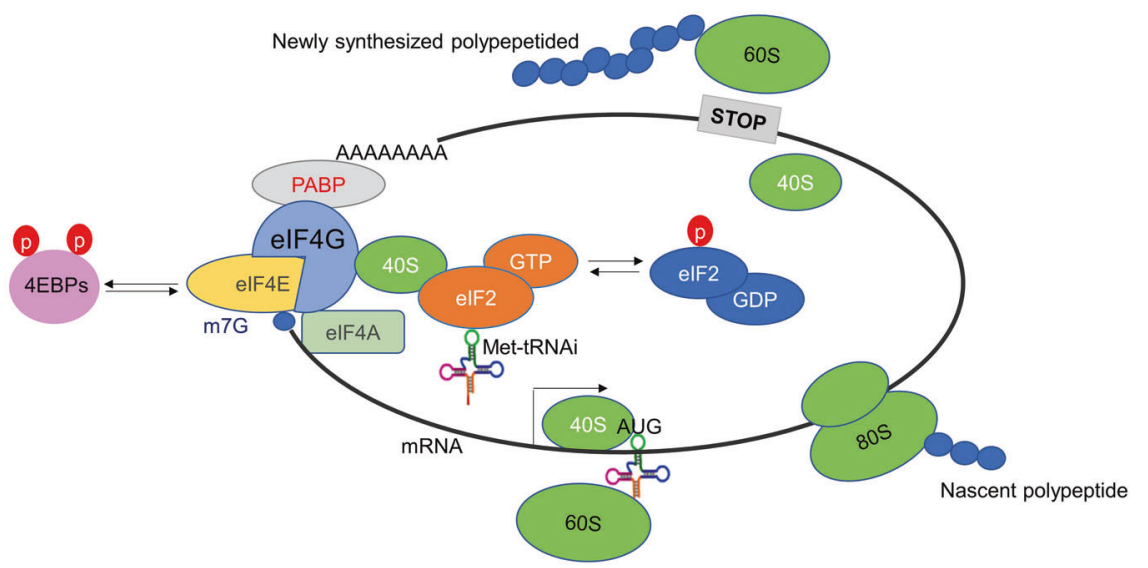

Fig. 2 The model of protein translation process. Translation is initiated by elF4F assembly, consists of elF4A, elF4E, and elF4G. The hypophosphorylated form of 4E-BPs competes with elF4G to bind elF4E, and phosphorylated elF2 $\alpha$ fails to recover the GTP required for MettRNAi, resulting in failure of the formation of elF4F complexes and inhibits translation. Extension: the initiation complex of cap-dependent translation scans the mRNA from the 5 'end to find the first start codon (AUG), and enters the ribosomal P-site via initiator methionyl tRNA (Met-tRNAi). Termination: when the mRNA with stop codon (UAG, UGA, UAA) enters the ribosomal A-site, it can't be recognized by activated amino acid (aa-tRNA), the translation process is finished 
growth of multiple cancer cell lines, ${ }^{30}$ highlighting the potential for targeting protein translation in the design of novel approaches in cancer treatment.

elF2a plays an important role in the process of translation initiation. Once phosphorylated, it loses its activity to recover the GTP required for Met-tRNAi, resulting in translation inhibition. Smad7 promotes the proliferation of colon cancer cells by activating elF2a to increase $\mathrm{CDC} 25$ protein translation, a phosphatase that dephosphorylates CDK2, controls progression of the cell cycle through the $S$ phase. ${ }^{31}$ In melanoma, the tumor suppressor gene PTEN increases the phosphorylation of elF2a via PDZ binding motif, promoting tumor cell apoptosis and inhibiting tumor proliferation. ${ }^{32}$ In prostate cancer, proteasome inhibitors (PIs) reduce the level of HIF1a protein translation by inducing elF2a phosphorylation, to inhibit the transcriptional activity of HIF1a, and downregulate the expression of target gene VEGF, thereby inhibiting angiogenesis. ${ }^{33}$ However, whether phosphorylated elF2a serving as protumor or antitumor role remains controversial, depending on the particular proteins affected on different tumor types and grades. There are studies indicating that increased elF2a phosphorylation may promote cancer development by enhancing the translation of target mRNAs. For example, decreased expression of PKR (also known as EIF2AK2), which is an elF2a phosphatase, is associated with less aggressive human cancers. ${ }^{8}$ GCN2 phosphorylates elF2a, promotes ATF4 protein translation by delayed restart (two uORFs in mRNA leader), ${ }^{34}$ which enhances tumorigenicity of fibrosarcoma cells in nude mice. ${ }^{35}$ Because of the paradoxical functions of elF2a, directly inhibiting phosphorylation need to consider the specific microenvironment of tumors.

\section{THE EFFECTS OF POST-TRANSCRIPTIONAL REGULATION ON PROTEIN TRANSLATION IN TUMORS}

RNA-binding protein

As the most widely studied post-translational regulators, RNAbinding proteins (RBPs) are involved in all aspects of RNA biological processes, including RNA metabolism, transport, and localization. Mechanistically, it has been speculated that RBPs reshape the structure of certain mRNA to change the affinity for translation machinery, thus affecting its translation. ${ }^{36}$ However, the exact mechanism still needs to be explored and confirmed. Functionally, they play a pivotal role in regulating the RNA translation in tumorigenesis ${ }^{37}$ (Table 1). For example, Musashi-1 (MSI1) and Musashi-2 (MSI2) are highly expressed in a variety of tumors, and they can functionally inhibit or promote the translation of both essential oncogenes and tumor suppressor genes through their $\mathrm{N}$-terminal RNA recognition sequence (RRM). ${ }^{38}$ Kawahara et al. found that MSI1 competed with elF4G to bind PABP to inhibit translation of downstream genes to maintain the stem cell status. ${ }^{39}$ Sun-Mi et al. found MSI2 controlled efficient translation of Hoxa9, Myc, and Ikzf2 mRNAs to maintain the mixed-lineage leukemia (MLL) self-renewal program. $^{40}$ In a research carried by Li and his colleagues, elF3 was highly expressed in liver cancer, playing an important role in the initiation of translation and promotes the development of cancer. ${ }^{41}$ In leukemia, SYNCRIP is a new RBP that promotes HOXA9 translation and controls myeloid leukemia stem cell programs. ${ }^{42}$ CELF1 (CUGBP Elav-like family member 1), inhibits MYC protein translation to reduce the renew and proliferation of small intestinal epithelial cells. ${ }^{43}$ In the colorectal cancer, HuR (ELAVlike RNA-binding protein 1), binds to the 5'UTR region of the proapoptotic gene caspase-2L, inhibiting its translation, and promoting tumor cell antiapoptosis activity. ${ }^{44}$ In RKO intestinal cancer cells of wild-type p53, under short-term UV irradiation, HuR can promote P53 translation. ${ }^{45}$ Moreover, HuR inhibits wnt-5a protein translation in breast tumors. ${ }^{46}$ Meanwhile, there are reports indicating that $\mathrm{CPEBs}$ (cytoplasmic polyadenylation element binding protein) induce cytoplasmic polyadenylation with specific polyA polymerase to enhancing translation and facilitating tumorigenesis and metastasis. ${ }^{47}$ IMP-3, a member of the insulin-like growth factor II (IGF-II) mRNA-binding protein (IMP) family, promotes IGF-II protein translation to induce the tumor cell proliferation in leukemia. ${ }^{48}$ When IFN- $\gamma$ is used to treat inflammation in bone marrow cells, GAIT complex inhibits VEGFA translation by binding to the $3^{\prime}$ UTR of VEGFA, while under hypoxic conditions, hnRNP L (heteroribonucleoprotein) is activated, interacting with DRBP76 (interleukin enhancer binding factor 3) and hnRNP A2/B1 to form a heterotrimer, which blocks GAIT-mediated translation inhibition, and promotes VEGFA expression. ${ }^{49}$ Based on these studies, it is evident that RBPs participate in tumor stemness, metastasis, proliferation, and immunity by influencing the translation of target genes. However, as RBPs are nonenzymatic, indicating the lack of hydrophobic pockets capable of binding small-molecule inhibitors, they are difficult to be therapeutic targets.

\section{Noncoding RNAs}

The noncoding RNA described in this review mainly includes small RNA (microRNA), long noncoding RNA (IncRNA), and circular RNA (circRNA), which have an important effect on tumors. ${ }^{50}$ In Table 2, we summarized the effects of noncoding RNA on translation procedure in tumors.

microRNA

MicroRNA is small noncoding RNA containing about 22 nucleotides. By complementary base pairing, microRNAs bind to the $3^{\prime} U T R$ region or $5^{\prime} U T R$ region of mRNA, thus mediating the degradation or translation of target mRNAs. ${ }^{9}$ It's widely recognized that miRNAs inhibit cap-dependent translation at the

Table 1. RNA-binding proteins involved in translation regulation

\begin{tabular}{llll}
\hline RBPs & Protein & Clinical correlates in cancers \\
\hline MSI1 & Inhibits downstream genes & Maintains the stem cell status \\
MSI2 & Promotes Hoxa9, Myc, and Ikzf2 & Maintains the self-renewal program of MLL \\
SYNCRIP & Promotes HOXA9 translation & Control myeloid leukemia stem cell program \\
CELF1 & Inhibits MYC translation & Inhibits the renew and proliferation of small intestinal epithelial cells & 44 \\
HUR & Inhibits Caspase-2L translation & Promotes antiapoptosis of the colorectal cancer cell \\
HUR & Promotes P53 translation & In intestinal cancer cells \\
CPEBs & Inhibits wnt-5a translation regulates polyadenylation & In breast cancer \\
IMP-3 & and resulting translation & Facilitates tumorigenesis and metastasis \\
GAIT & promotes IGF-II translation & Promotes tumor proliferation in leukemia \\
& inhibits VEGFA translation & Bone marrow cells under immunotherapy \\
\hline
\end{tabular}


Table 2. Noncoding RNAs involved in translation regulation

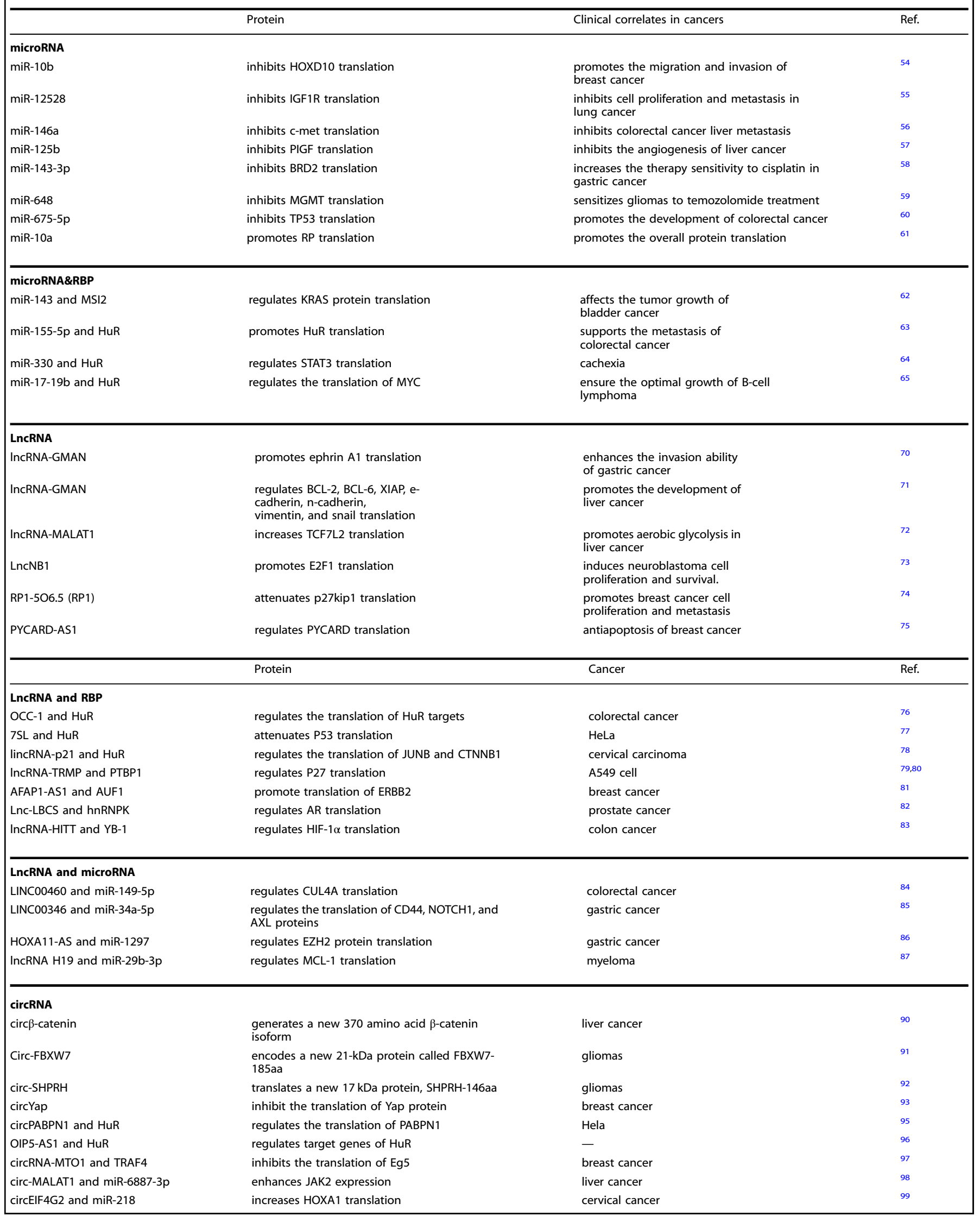


initiation step by dissociating the translation initiation factors from targeted mRNAs. ${ }^{51,52}$ Over the past decades, a bunch of researches demonstrated that microRNA plays an important role in tumorigenesis and development. In terms of influencing the translation process alone, microRNA-10b (miR-10b) is highly expressed in metastatic breast cancer. miR-10b inhibits HOXD10 translation via interaction with its $3^{\prime}$ UTR and promotes the migration and invasion. ${ }^{53}$ miR-12528 inhibits insulin-like growth factor 1 receptor (IGF1R) translation to inhibit cell proliferation and metastasis in lung cancer. ${ }^{54}$ miR-146a inhibits tumor formation, and colorectal cancer liver metastasis by limiting c-Met translation. ${ }^{55}$ The expression of miR-125b is low in liver cancer tissue comparing to normal liver tissue, and overexpression miR-125b can inhibit the translation of placental growth factor (PIGF) protein to reduce the angiogenesis. ${ }^{56}$ Comparing to normal gastric tissue, the expression of mir-143-3p is significantly lower in gastric cancer tissues. Overexpression of mir-143-3p inhibits BRD2 protein translation, thereby inhibiting cancer cell proliferation and increasing the therapy sensitivity to cisplatin. ${ }^{57}$ In glioma, miR648 inhibits 06-methylguanine-DNA methyltransferase (MGMT) protein translation, reducing the production of MGMT and thus sensitizing MGMT-expressing gliomas to temozolomide treatment. $^{58}$ In colorectal cancer, overexpressed prostaglandin E2 (PGE2) upregulates miR-675-5p expression to inhibits TP53 translation, thus promoting tumor development. ${ }^{59}$ In a research carried out by Orom et al., miR-10a could bind to the $5^{\prime}$ UTR of ribosomal protein (RP) mRNA to promote the translation under amino acid starvation conditions, and regulated the overall protein translation. ${ }^{60}$ In conclusion, microRNAs can inhibit the proliferation, metastasis, angiogenesis of tumors and sensitize tumors to chemotherapy drugs by decreasing the protein synthesis of certain target genes.

MicroRNAs can also interact with RBPs to jointly regulate protein translation. For example, miR-143 inhibits MSI2 protein translation through $3^{\prime}$ UTR base pairing to reduce the translation of KRAS which interacts with $\mathrm{MSI} 2$, thereby inhibiting tumor growth in bladder cancer. ${ }^{61}$ In colorectal cancer, MiR-155-5p promotes HuR translation to support cell metastasis. ${ }^{62}$ In tumor-induced cachexia, HuR promotes translation of STAT3 mRNA by preventing miR-330mediated translation inhibition. ${ }^{63}$ In B-cell lymphoma, miR-17-19b indirectly reduces the translation efficiency of MYC by downregulating Chek2 and increasing the binding of HuR to MYC mRNA. ${ }^{64}$

Taken together, these studies highlight the balance between RBPs and microRNAs has an important impact on the translation of target genes. Indeed, antitumor therapeutics targeting microRNAs by miRNA mimics and inhibitors have shown promise in preclinical development.

\section{LncRNA}

LncRNAs contain about 200 nucleotides, ${ }^{65}$ and play an important role in tumor progression by regulating the chromatin organization, transcription, mRNA stability, protein translation, and posttranslational modification. ${ }^{66-68}$ As the scaffold of nuclear bodies involved in the translation regulation, IncRNAs play a crucial part in gene expression.

Gastric cancer metastasis-associated long noncoding RNA (GMAN) promotes the translation of ephrin $A 1$ by binding with antisense GMAN RNA (GMAN-AS), enhancing the invasion ability of gastric cancer cells. ${ }^{69}$ Furthermore, it is highly expressed in liver cancer and interacts with elF4B. By inhibiting the dephosphorylation of PPP2R2A, it promotes phosphorylation of serine 422 at eIF4B, and subsequent phosphorylated elF4B increases the antiapoptotic proteins $\mathrm{BCL}-2, \mathrm{BCL}-6, \mathrm{XIAP}$ and migration-related proteins e-cadherin, $\mathrm{n}$-cadherin, vimentin, and snail translation to promote the development of liver cancer. ${ }^{70}$ IncRNA-MALAT1 increases TCF7L2 translation by activating the mTOR-4EBP1 signal axis and SRSF1, and promotes aerobic glycolysis in liver cancer. ${ }^{71}$ LncNB1 is overexpressed in MYCN-amplified neuroblastoma and induces neuroblastoma cell proliferation and survival. In mechanism, IncNB1 promotes the transcription of DEPDC1B and translation of E2F1 mRNA, leading to phosphorylation of N-MYC and stable protein expression. ${ }^{72}$ RP1-506.5 (RP1) is highly expressed in breast cancer and promotes the proliferation and metastasis of breast cancer cell. The combination of RP1 and p-4E-BP1/elF4E can prevent elF4E from interacting with elF4G, thus attenuating the translation of p27kip1 mRNA, and increasing the expression of snail to improve the invasiveness. ${ }^{73}$ In breast cancer cells, PYCARD-AS1, the antisense IncRNA of proapoptotic gene PYCARD, overlaps and interacts with PYCARD at the $5^{\prime}$ end, inhibiting ribosome assembly in the cytoplasm to limit the translation efficiency of PYCARD. ${ }^{74}$

LncRNAs also play a role in protein translation by interacting with RBPs. LncRNA OCC-1 plays as suppressor in colorectal cancer. By binding to the E3 ligase $\beta$-TRCP, it increases the ubiquitination of HuR, which leads to a decrease in the expression of downstream genes. ${ }^{75}$ LncRNA 7SL, highly expressed in tumor cells, attenuates the translation of the tumor suppressor protein P53 by binding to the $3^{\prime}$ UTR of P53 mRNA and squeezing HuR binding. ${ }^{76}$ On the other hand, HuR recruits let-7/Ago2 to IncRNAp21, reducing its stability. The decreased binding of IncRNA-p21 to mRNAs of JUNB and CTNNB1 promotes their protein translation in HeLa cells. ${ }^{77}$ LncRNA-TRMP promotes cancer cell proliferation and G1/S cycle progression when P53 is activated, the authors test the cycle-related proteins and find that TRMP negatively regulates P27. By inhibiting PTBP1, P27 mRNA-binding protein, TRMP ultimately inhibits IRES-dependent translation of $\mathrm{P} 27$ protein. $^{78,79}$ In terms of chemotherapy response, the expression level of actin filament-related protein 1 antisense RNA 1 (AFAP1-AS1) is higher in trastuzumab-resistant cells than that in sensitive cells. Exosomal AFAP1-AS1 can induce trastuzumab resistance. Mechanically, AFAP1-AS1 interacts with the AUF1 to promote translation of ERBB2, which is used to represent the RNA encoding the HER-2 protein. ${ }^{80}$ Lnc-LBCS expression is low in castration-resistant prostate cancer (CRPC) cell lines and tissues. In the absence of androgens, knockdown of LBCS is sufficient to activate androgen receptor (AR) signaling, consequently increasing $A R$ protein translation. In terms of mechanism, LBCS directly interacts with hnRNPK to inhibit the form of hnRNPK-AR complex and thus reduces AR translation. ${ }^{81}$ The low expression of HITT (translation level HIF-1a inhibitor) is related to the advanced stage of colon cancer. After the expression is restored, HITT inhibits tumor angiogenesis and tumor growth. Mechanistically, the direct binding of HITT and YB-1 deceives and prevents YB-1 from binding to $5^{\prime}$ UTR of HIF-1 a mRNA, which relieves the translation promotion effect of YB-10n HIF-1a. 82

In addition, LncRNAs can also serve as competitive endogenous RNAs (ceRNAs) to sponge or decoy on microRNAs to regulate protein translation. For example, LINC00346 recruits argonaute 2 (Ago2), which acts as a molecular sponge, antagonizes miR-34a-5p to inhibit the translation of CD44, NOTCH1, and AXL proteins, and promotes the development of gastric cancer. ${ }^{83}$ LINC00460 is highly expressed in colorectal cancer cells. As ceRNA, it antagonizes miR$149-5 p$ to inhibit cullin 4A (CUL4A) translation and affects cell growth and apoptosis. ${ }^{84}$ HOXA11-AS acts as a sponge of miR-1297, antagonizing its ability to inhibit the translation of EZH2 protein and increasing EZH2 protein into the nucleus to inhibit the transcription of PRSS8 and KLF2. In conclusion, HOXA11-AS/miR$1297 / E Z H 2$ crosstalk promotes the proliferation, migration and invasion of gastric cancer cell. ${ }^{85}$ LncRNA H19 is highly expressed in multiple myeloma and promotes tumor cell growth and bortezomib resistance via inhibiting miR-29b-3p expression and enhancing $\mathrm{MCL}-1$ translation. ${ }^{86}$

circRNA

With the development of high-throughput transcriptome sequencing and computational methods, a bunch of researches reveal 


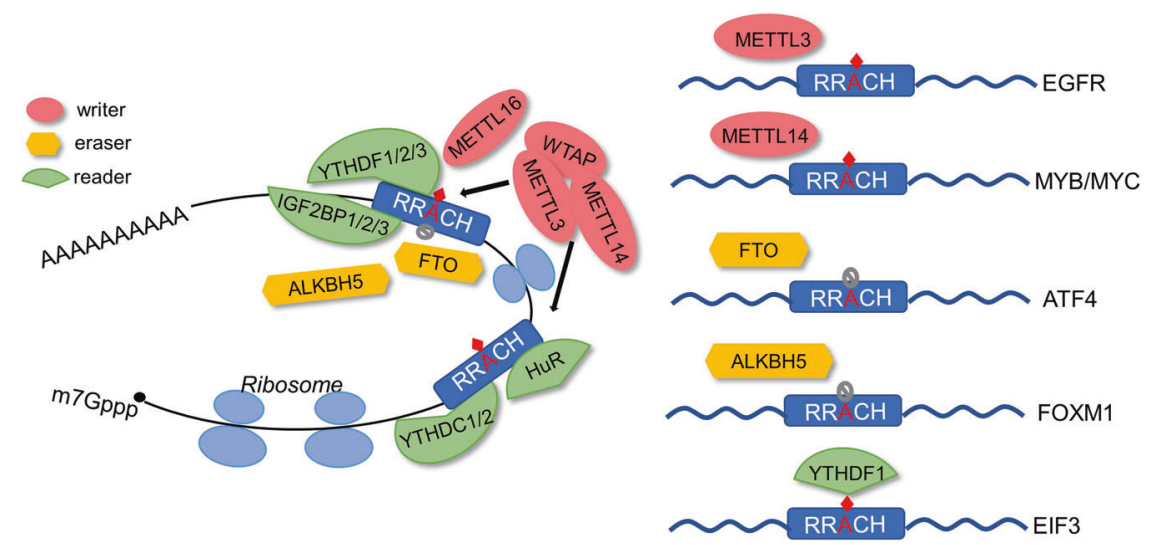

Fig. $3 \mathrm{~m}^{6} \mathrm{~A}$ modification regulates protein translation. The $\mathrm{m}^{6} \mathrm{~A}$ modification is catalyzed by the writers (METTL3/METTL14/WTAP, WETTL16) and demethylated by erasers (FTO, ALKBH5). The $\mathrm{m}^{6} \mathrm{~A}$ modification is recognized by the readers (YTHDF1/2/3, YTHDC1/2, IGF2BP1/2/3, and $\mathrm{HuR}$ ). The translation procedure of target genes can be influenced by the m6A modification level

that a large amount of circular RNAs (circRNAs) are endogenous, conservative, and stable in eukaryotic cells. ${ }^{87}$ CircRNAs are a new type of noncoding RNA, characterized with covalent closed loop formed by reverse splicing of precursor mRNA. Recent studies have demonstrated the relation between circRNAs and polysomes. Some circRNAs contain the start codon AUG and the recognized open reading frame (ORF), indicating that circRNAs have a potential protein coding function. ${ }^{88}$ Circ $\beta$-catenin, abundantly expressed in liver cancer, generates a 370 amino acid $\beta$-catenin isoform, which utilizes the start codon as linear $\beta$-catenin RNA transcription and translation ends with a new termination caused by circularization codon. This newly born $\beta$-catenin can resist GSK3 phosphorylation, and finally stabilize expression and activate WNT signaling pathway. ${ }^{89}$ Circ-FBXW7 is highly expressed in the normal human brain. It relies on the IRES to cross the open reading frame and encode a $21 \mathrm{kDa}$ protein, FBXW7-185aa. In gliomas, the upregulation of FBXW7-185aa inhibits proliferation and cell cycle. ${ }^{90}$ In addition, circ-SHPRH can also translate a $17 \mathrm{kDa}$ protein, SHPRH-146aa, through IRES-driven open reading frames (ORF), and both are low expressed in gliomas. Overexpression of them can inhibit gliomas development. ${ }^{91}$

In addition to being able to regulate translation by itself, circRNAs can also regulate other protein translation by regulating translation initiation. For example, overexpression of ciri-Yap inhibits the assembly of the translation initiation complex (elF4G and PABP), thus inhibiting the translation of Yap protein. As a consequence, overexpression of circYap inhibits the proliferation, migration, and colony formation of breast cancer cells. ${ }^{92}$. Furthermore, circRNAs interact with RBPs or act as ceRNA through sponge microRNAs to affect translation and play an important role in tumorigenesis and development. ${ }^{93}$ In HeLa cells, circPABPN1 (hsa_circ_0031288) prevents HuR from binding to PABPN1 mRNA and inhibits the translation of PABPN1 protein. ${ }^{94}$ Similarly, OIP5AS1 inhibits tumor cell proliferation by preventing HuR from binding to target genes. ${ }^{95}$ In breast cancer, circRNA-MTO1 (hsa-circRNA-007874) binds to the tumor necrosis factor receptor-associated factor 4 (TRAF4), thereby inhibiting the translation of mitogenic kinesin (Eg5) and reversing Monastrol (kinesin inhibitor) resistance. ${ }^{96}$ circ-MALAT1 combines with ribosomes and tumor suppressor gene PAX5 to form a complex, hindering the translation of PAX5 and helping maintain cancer stem cells characteristics. It is also found that circ-MALAT1 promotes liver cancer stem cell self-renewal by acting as the miR-6887-3p sponge, enhancing JAK2 expression and activating the JAK2/STAT3 signaling pathway. ${ }^{97}$ In cervical cancer, highly expressed circEIF4G2 acts as a sponge for miR-218 which promotes HOXA1 protein translation, and consequently promotes cancer cell proliferation and migration. ${ }^{98}$ 6-methyladenine (m6A) modification

The m6A modification of RNA, as the most common mRNA modification in mammalian cells, usually occurs at the consensus sequence $5^{\prime}-\mathrm{RRACH}-3$ ' $(\mathrm{R}=\mathrm{A}$ or $\mathrm{G} ; \mathrm{H}=\mathrm{A} / \mathrm{C} / \mathrm{U}){ }^{99} \mathrm{~A}$ bunch of recent studies about m6A modification have been performed in tumors. The m6A modification of RNA mainly consists of three parts: methyltransferase complex (writer), demethyltransferase (eraser) and recognition protein (reader). Writer mainly consists of methyltransferase 3 (METTL3), methyltransferase 14 (METTL14) and Wilms tumor 1-associating protein (WTAP). ${ }^{100}$ Recently, METTL16, KIAA1429, RNA-binding motif protein 15 (RBM15) and Zinc finger CCC domain protein 13 ( $\mathrm{ZC} 3 \mathrm{H} 13)$ also have been found to have methyltransferase activity. ${ }^{101,102}$ The eraser mainly consists of FTO and ALKBH5. These two genes reversibly regulate the m6A level of RNA. Reader proteins mainly include YTHDF1/2/3, YTHDC1/2, and some RNA-binding proteins, including insulin-like growth factor 2 binding protein 1/2/3 (IGF2BP1/2/3), RNA-bound ELAV protein 1 (ELAVL1, also known as HuR), heterogeneous ribonucleoproteins (HNRNPs), FMR1 (Fragile X mental retardation 1) (Fig. 3).

As the most extensive post-transcriptional modification of RNA, m6A affects the splicing, stability, export and translation of RNA. ${ }^{103}$ Thus, m6A modification of RNA has a huge impact on the protein translation process of tumor cells. ${ }^{15,102,104}$ As the main methyltransferase, METTL3 stimulates the initiation of translation by increasing the recovery of elF3, resulting in translation of target genes including epidermal growth factor receptor (EGFR) and Hippo pathway effector TAZ. ${ }^{105}$ METTL14, a key component of the m6A methyltransferase complex, is highly expressed in acute myeloid leukemia (AML). METTL14 increases m6A modification of MYB/MYC, promotes the protein translation, and maintains AML stem/initial cell self-renewal. ${ }^{106}$ In response to stress, such as amino acid starvation, the retranslation initiation of ATF4 is not mediated by the elF2a signal but relies on m6A modification in the $5^{\prime}$ UTR, which can control ribosome scanning and subsequent selection of start codons. Consistently, demethyltransferase FTO promotes ATF4 protein translation. ${ }^{104} \mathrm{~A}$ long noncoding RNA antisense to FOXM1 (FOXM1-AS) promotes the interaction of ALKBH5 with FOXM1 mRNA, increased FOXM1 expression by demethylation leading to the proliferation of stem-like cells in glioblastoma. ${ }^{107}$ YTHDF1 recognizes $\mathrm{m} 6 \mathrm{~A}$ modified $\mathrm{mRNA}$. High-throughput sequencing showed that the ribosomes loaded by YTHDF1 targeted RNA are directly proportional to the binding of YTHDF1. Furthermore, YTHDF1 interacts with translation initiation factors to directly accelerate translation of ribosomal-bound mRNA. ${ }^{103,108,109}$ For instance, Tao and his colleagues found the translation of EIF3C, a subunit of translation initiation factor EIF3, was increased by YTHDF1 in an $\mathrm{m}^{6} \mathrm{~A}$-dependent manner. Consequently, the overall translation output of ovarian cancer is triggered, accelerating the 
tumorigenesis and metastasis. ${ }^{110}$ In HeLa cells, sequencing results combined with experiments have shown that YTHDF3 can coordinate with YTHDF1 to regulate translation. The mechanisms include YTHDF3's regulation on translation initiation without directly binding translation initiation factors, and cooperation with YTHDF1 to jointly regulate translation. ${ }^{111,112}$

In addition, leucine-rich pentapeptide repeat (LRPPRC) may also act as $\mathrm{m} 6 \mathrm{~A}$ recognition protein, ${ }^{102}$ affects mitochondrial protein translation. ${ }^{113}$ In fragile $X$ syndrome, $X$ fragile mental retardation protein (FMRP) inhibits translation by binding to the $L 5$ protein on the $80 \mathrm{~S}$ ribosome directly. ${ }^{114}$ In autism spectrum disorders, FMR1 affects RNA metabolism by recognizing $\mathrm{m} 6 \mathrm{~A}$ modifications, including protein translation. ${ }^{115} \mathrm{M} 6 \mathrm{~A}$ modification can also affect tRNA conditions and translation extension steps, thus altering translation kinetics. ${ }^{116}$ However, the exact mechanism of these genes has not been studied in tumors. Most of the m6A modifications of mRNA and small noncoding RNAs depend on the sequences and structures of mRNAs to work, and they will inevitably have a competitive or cooperative relationship in the translation process of mRNAs.

Collectively, the abnormal regulation of m6A methylation is closely related with the development of cancers ranging from metabolism, cell self-renewal, differentiation, and metastasis. Increasing evidences show that m6A modification of mRNA plays a dual role in cancer. On the one hand, methyltransferase complex increases the m6A modification of oncogenes to promote cancer development. On the other hand, FTO and ALKBH5 also promote tumor progression by reducing the modification of target genes. ${ }^{17,118}$ Therefore, the exact role of $\mathrm{m}^{6} \mathrm{~A}$ in tumors needs to be judged according to the cellular contexts.

The effects of codons and tRNAs on protein translation in tumors The factors discussed above, including RBPs, noncoding RNAs and RNA m6A modification, perform function by regulating the mRNA structure and translation initiation complex. In addition to those factors, codons are one of the factors that affect the translation extension. Different codons were translated at different speeds. ${ }^{119}$ For example, GAA was found translated with a rate of 21.6 codons while GAG at 6.4 codons per second. ${ }^{120}$ Codon extension mainly depends on the entry of tRNA into the ribosome A-site through anticodon recognition. ${ }^{121}$ Therefore, the abundance of tRNA also plays a role in translation efficiency. Growing evidence demonstrated that dysregulation of tRNA was involved in tumor progression. Compared with normal cells, breast cancer cells have a 3-fold increase in nuclear-encoded tRNA expression and a 5-fold increase in mitochondrial-encoded tRNA to facilitate the translation of a subset of regulatory genes. ${ }^{122}$ For example, tRNAGluUUC and tRNAArgCCG were upregulated in breast cancer and promoted metastasis by increasing the ribosome occupancy of transcripts enriched for their cognate codons. ${ }^{123}$ Further researches identified two different subsets of tRNA pool between proliferation and differentiation, of which one is induced in proliferating cells and inhibited in other cases, and the other exhibiting the opposite characteristics. Subsequently, if this program hijacked by tumor cells to selectively upregulate proliferative tRNA, it will likely promote the translation of precancerous transcripts. ${ }^{124}$ In the future, tRNA may serve as a biomarker for cancer.

\section{CONCLUSION}

Translation is an essential procedure for the protein expression, in this review we summarized the common factors affecting this procedure, including translation initiation factors, RBPs, noncoding RNAs (microRNAs, IncRNAs and circRNAs), and RNA m6A modification (Fig. 1). In addition, codons and tRNAs play important role on the translation elongation rate. The past decades have witnessed the rapid development of experimental techniques for research of translation, such as isotope or puromycin labeling, ${ }^{125-128}$ polysome profiling experiments using ultracentrifugation and density gradient separation, etc., ${ }^{129-131}$ and these up-to-date techniques paved a wider path for researchers to investigate the potential molecular mechanism of tumor biology. After detecting abnormal translation in tumorigenesis induced by the above influencing elements, m6A and noncoding RNAs may potentially contribute to the prediction of the prognosis of tumor patients and the clinical application of antitumor therapy. Because of the widespread existence of RBPs, noncoding RNAs, m6A modification and tRNAs in eukaryotic cell, clarifying their relationship with aberrant protein translation is of great significance for the tumor treatment.

\section{ACKNOWLEDGEMENTS}

This work was supported by National Natural Science Foundation of China (91740106; 81772944; 81672723; 81672360).

\section{ADDITIONAL INFORMATION}

Competing interests: The authors declare no competing interests.

\section{REFERENCES}

1. Siegel, R. L., Miller, K. D. \& Jemal, A. Cancer statistics, 2019. CA Cancer J. Clin. 69 7-34 (2019).

2. Stillman, B. Histone modifications: insights into their influence on gene expression. Cell 175, 6-9 (2018).

3. Bourgeois, C. F., Mortreux, F. \& Auboeuf, D. The multiple functions of RNA helicases as drivers and regulators of gene expression. Nat. Rev. Mol. Cell Biol. 17, 426-438 (2016).

4. Darzynkiewicz, E. et al. Beta-globin mRNAs capped with m7G, m2.7(2)G or $\mathrm{m} 2.2 .7(3) \mathrm{G}$ differ in intrinsic translation efficiency. Nucleic Acids Res. 16, 8953-8962 (1988).

5. Xu, F. \& Cohen, S. N. RNA degradation in Escherichia coli regulated by $3^{\prime}$ adenylation and $5^{\prime}$ phosphorylation. Nature 374, 180-183 (1995).

6. Tudek, A., Lloret-Llinares, M. \& Jensen, T. H. The multitasking polyA tail: nuclear RNA maturation, degradation and export. Philos. Trans. R. Soc. Lond. B Biol. Sci. 373, (2018) https://doi.org/10.1098/rstb.2018.0169.

7. Mowen, K. A. \& David, M. Unconventional post-translational modifications in immunological signaling. Nat. Immunol. 15, 512-520 (2014).

8. Silvera, D., Formenti, S. C. \& Schneider, R. J. Translational control in cancer. Nat. Rev. Cancer 10, 254-266 (2010).

9. Spriggs, K. A., Bushell, M. \& Willis, A. E. Translational regulation of gene expression during conditions of cell stress. Mol. Cell. 40, 228-237 (2010).

10. Bhat, M. et al. Targeting the translation machinery in cancer. Nat. Rev. Drug Discov. 14, 261-278 (2015).

11. Hershey, J. W. B., Sonenberg, N. \& Mathews, M. B. Principles of translational control. Cold Spring Harb. Perspect. Biol. 11, (2019) https://doi.org/10.1101/ cshperspect.a032607.

12. Jackson, R. J., Hellen, C. U. \& Pestova, T. V. The mechanism of eukaryotic translation initiation and principles of its regulation. Nat. Rev. Mol. Cell Biol. 11, 113-127 (2010).

13. Hellen, C. U. T.Translation termination and ribosome recycling in Eukaryotes. Cold Spring Harb. Perspect. Biol. 10, (2018) https://doi.org/10.1101/cshperspect. a032656.

14. Jung, H., Gkogkas, C. G., Sonenberg, N. \& Holt, C. E. Remote control of gene function by local translation. Cell 157, 26-40 (2014).

15. Frye, M., Harada, B. T., Behm, M. \& He, C. RNA modifications modulate gene expression during development. Science 361, 1346-1349 (2018).

16. Dever, T. E., Dinman, J. D. \& Green, R. Translation elongation and recoding in Eukaryotes. Cold Spring Harb. Perspect. Biol. 10, (2018) https://doi.org/10.1101/ cshperspect.a032649.

17. Pogue-Geile, K. et al. Ribosomal protein genes are overexpressed in colorectal cancer: isolation of a cDNA clone encoding the human S3 ribosomal protein. Mol. Cell Biol. 11, 3842-3849 (1991).

18. Williamson, D. et al. Nascent pre-rRNA overexpression correlates with an adverse prognosis in alveolar rhabdomyosarcoma. Genes Chromosomes Cancer 45, 839-845 (2006).

19. White, R. J. et al. Repression of RNA polymerase III transcription by the retinoblastoma protein. Nature 382, 88-90 (1996).

20. Presnyak, V. et al. Codon optimality is a major determinant of mRNA stability. Cell 160, 1111-1124 (2015). 
21. Bjornsti, M. A. \& Houghton, P. J. Lost in translation: dysregulation of capdependent translation and cancer. Cancer Cell. 5, 519-523 (2004).

22. Boussemart, L. et al. elF4F is a nexus of resistance to anti-BRAF and anti-MEK cancer therapies. Nature 513, 105-109 (2014).

23. Truitt, M. L. et al. Differential requirements for elF4E dose in normal development and cancer. Cell 162, 59-71 (2015).

24. Mizutani, R. et al. Oncofetal protein IGF2BP3 facilitates the activity of protooncogene protein elF4E through the destabilization of EIF4E-BP2 mRNA. Oncogene 35, 3495-3502 (2016).

25. $\mathrm{Xu}, \mathrm{Y}$. et al. Translation control of the immune checkpoint in cancer and its therapeutic targeting. Nat. Med. 25, 301-311 (2019).

26. Graff, J. R. et al. Therapeutic suppression of translation initiation factor elF4E expression reduces tumor growth without toxicity. J. Clin. Invest. 117, 2638-2648 (2007).

27. Reich, S. H. et al. Structure-based design of pyridone-aminal eFT508 targeting dysregulated translation by selective mitogen-activated protein kinase interacting kinases 1 and 2 (MNK1/2) inhibition. J. Med. Chem. 61, 3516-3540 (2018).

28. Dowling, R. J. et al. mTORC1-mediated cell proliferation, but not cell growth, controlled by the 4E-BPs. Science 328, 1172-1176 (2010).

29. Martineau, Y., Azar, R., Bousquet, C. \& Pyronnet, S. Anti-oncogenic potential of the elF4E-binding proteins. Oncogene 32, 671-677 (2013).

30. Moerke, N. J. et al. Small-molecule inhibition of the interaction between the translation initiation factors elF4E and elF4G. Cell 128, 257-267 (2007).

31. Stolfi, C. et al. A functional role for Smad7 in sustaining colon cancer cell growth and survival. Cell Death Dis. 5, e1073 (2014).

32. Mounir, Z. et al. Tumor suppression by PTEN requires the activation of the PKRelF2alpha phosphorylation pathway. Sci. Signal. 2, ra85 (2009).

33. Zhu, K. et al. Control of HIF-1alpha expression by elF2 alpha phosphorylationmediated translational repression. Cancer Res. 69, 1836-1843 (2009).

34. Pathak, S. S. et al. The elF2alpha kinase GCN2 modulates period and rhythmicity of the circadian clock by translational control of Atf4. Neuron 104, 724-735 (2019). e726.

35. Kozel, C. et al. Overexpression of elF5 or its protein mimic 5MP perturbs elF2 function and induces ATF4 translation through delayed re-initiation. Nucleic Acids Res. 44, 8704-8713 (2016).

36. Martinez-Salas, E. et al. RNA-binding proteins impacting on internal initiation of translation. Int. J. Mol. Sci. 14, 21705-21726 (2013).

37. Zhang, B. et al. A comprehensive expression landscape of RNA-binding proteins (RBPs) across 16 human cancer types. RNA Biol. 17, 211-226 (2020).

38. Kudinov, A. E., Karanicolas, J., Golemis, E. A. \& Boumber, Y. Musashi RNA-binding proteins as cancer drivers and novel therapeutic targets. Clin. Cancer Res. 23, 2143-2153 (2017).

39. Kawahara, H. et al. Neural RNA-binding protein Musashi1 inhibits translation initiation by competing with elF4G for PABP. J. Cell Biol. 181, 639-653 (2008).

40. Park, S. M. et al. Musashi2 sustains the mixed-lineage leukemia-driven stem cell regulatory program. J. Clin. Invest. 125, 1286-1298 (2015).

41. Li, T. et al. Transcriptomic analyses of RNA-binding proteins reveal elF3c promotes cell proliferation in hepatocellular carcinoma. Cancer Sci. 108, 877-885 (2017).

42. $\mathrm{Vu}$, L. P. et al. Functional screen of MSI2 interactors identifies an essential role for SYNCRIP in myeloid leukemia stem cells. Nat. Genet. 49, 866-875 (2017).

43. Liu, L. et al. Competition between RNA-binding proteins CELF1 and HuR modulates MYC translation and intestinal epithelium renewal. Mol. Biol. Cell. 26, 1797-1810 (2015).

44. Winkler, C. et al. Attenuation of the ELAV1-like protein HuR sensitizes adenocarcinoma cells to the intrinsic apoptotic pathway by increasing the translation of caspase-2L. Cell Death Dis. 5, e1321 (2014).

45. Mazan-Mamczarz, K. et al. RNA-binding protein HuR enhances p53 translation in response to ultraviolet light irradiation. Proc. Natl Acad. Sci. USA 100, 8354-8359 (2003).

46. Leandersson, K., Riesbeck, K. \& Andersson, T. Wnt-5a mRNA translation is suppressed by the Elav-like protein HuR in human breast epithelial cells. Nucleic Acids Res. 34, 3988-3999 (2006)

47. D'Ambrogio, A., Nagaoka, K. \& Richter, J. D. Translational control of cell growth and malignancy by the CPEBs. Nat. Rev. Cancer 13, 283-290 (2013).

48. Liao, B., Hu, Y., Herrick, D. J. \& Brewer, G. The RNA-binding protein IMP-3 is a translational activator of insulin-like growth factor II leader-3 mRNA during proliferation of human K562 leukemia cells. J. Biol. Chem. 280, 18517-18524 (2005).

49. Yao, P. et al. The HILDA complex coordinates a conditional switch in the $3^{\prime}-$ untranslated region of the VEGFA mRNA. PLoS Biol. 11, e1001635 (2013).

50. Beermann, J., Piccoli, M. T., Viereck, J. \& Thum, T. Non-coding RNAs in development and disease: background, mechanisms, and therapeutic approaches. Physiol. Rev. 96, 1297-1325 (2016).
51. Humphreys, D. T., Westman, B. J., Martin, D. I. \& Preiss, T. MicroRNAs control translation initiation by inhibiting eukaryotic initiation factor $4 \mathrm{E} /$ cap and poly(A) tail function. Proc. Natl Acad. Sci. USA 102, 16961-16966 (2005).

52. Duchaine, T. F. \& Fabian, M. R. Mechanistic insights into MicroRNA-mediated gene silencing. Cold Spring Harb. Perspect. Biol. 11, (2019) https://doi.org/ 10.1101/cshperspect.a032771.

53. Ma, L., Teruya-Feldstein, J. \& Weinberg, R. A. Tumour invasion and metastasis initiated by microRNA-10b in breast cancer. Nature 449, 682-688 (2007).

54. Jeon, S. H. et al. The novel hsa-miR-12528 regulates tumourigenesis and metastasis through hypo-phosphorylation of AKT cascade by targeting IGF-1R in human lung cancer. Cell Death Dis. 9, 493 (2018).

55. Bleau, A. M. et al. miR-146a targets c-met and abolishes colorectal cancer liver metastasis. Cancer Lett. 414, 257-267 (2018).

56. Alpini, G. et al. Regulation of placenta growth factor by microRNA-125b in hepatocellular cancer. J. Hepatol. 55, 1339-1345 (2011).

57. Chen, Z. et al. Integrated analysis of mouse and human gastric neoplasms identifies conserved microRNA networks in gastric carcinogenesis. Gastroenterology 156, 1127-1139 (2019). e1128.

58. Kreth, S. et al. In human glioblastomas transcript elongation by alternative polyadenylation and miRNA targeting is a potent mechanism of MGMT silencing. Acta Neuropathol. 125, 671-681 (2013).

59. Cen, B. et al. Prostaglandin E2 induces miR675-5p to promote colorectal tumor metastasis via modulation of p53 expression. Gastroenterology 158, 971-984. e10 (2019).

60. Orom, U. A., Nielsen, F. C. \& Lund, A. H. MicroRNA-10a binds the $5^{\prime}$ UTR of ribosomal protein mRNAs and enhances their translation. Mol. Cell. 30, 460-471 (2008).

61. Tsujino, T. et al. MicroRNA-143/Musashi-2/KRAS cascade contributes positively to carcinogenesis in human bladder cancer. Cancer Sci. 110, 2189-2199 (2019).

62. Al-Haidari, A. et al. MiR-155-5p controls colon cancer cell migration via posttranscriptional regulation of Human Antigen R (HuR). Cancer Lett. 421, 145-151 (2018).

63. Mubaid, S. et al. HuR counteracts miR-330 to promote STAT3 translation during inflammation-induced muscle wasting. Proc. Natl Acad. Sci. USA 116, 17261-17270 (2019).

64. Mihailovich, M. et al. miR-17-92 fine-tunes MYC expression and function to ensure optimal B cell lymphoma growth. Nat. Commun. 6, 8725 (2015).

65. Quinn, J. J. \& Chang, H. Y. Unique features of long non-coding RNA biogenesis and function. Nat. Rev. Genet. 17, 47-62 (2016).

66. Kopp, F. \& Mendell, J. T. Functional classification and experimental dissection of long noncoding RNAs. Cell 172, 393-407 (2018).

67. Schmitt, A. M. \& Chang, H. Y. Long noncoding RNAs in cancer pathways. Cancer Cell. 29, 452-463 (2016).

68. Yao, R. W., Wang, Y. \& Chen, L. L. Cellular functions of long noncoding RNAs. Nat. Cell Biol. 21, 542-551 (2019).

69. Zhuo, W. et al. Long noncoding RNA GMAN, up-regulated in gastric cancer tissues, is associated with metastasis in patients and promotes translation of ephrin A1 by competitively binding GMAN-AS. Gastroenterology 156, 676-691 (2019).

70. $\mathrm{Xu}$, J. et al. Long noncoding RNA GMAN promotes hepatocellular carcinoma progression by interacting with elF4B. Cancer Lett. 473, 1-12 (2020).

71. Malakar, P. et al. Long noncoding RNA MALAT1 regulates cancer glucose metabolism by enhancing mTOR-mediated translation of TCF7L2. Cancer Res. 79, 2480-2493 (2019).

72. Liu, P. Y. et al. The long noncoding RNA IncNB1 promotes tumorigenesis by interacting with ribosomal protein RPL35. Nat. Commun. 10, 5026 (2019).

73. Jia, X. et al. KLF5 regulated IncRNA RP1 promotes the growth and metastasis of breast cancer via repressing p27kip1 translation. Cell Death Dis. 10, 373 (2019).

74. Miao, H. et al. A long noncoding RNA distributed in both nucleus and cytoplasm operates in the PYCARD-regulated apoptosis by coordinating the epigenetic and translational regulation. PLoS Genet. 15, e1008144 (2019).

75. Lan, Y. et al. Long noncoding RNA OCC-1 suppresses cell growth through destabilizing HuR protein in colorectal cancer. Nucleic Acids Res. 46, 5809-5821 (2018).

76. Abdelmohsen, K. et al. 7SL RNA represses p53 translation by competing with HuR. Nucleic Acids Res. 42, 10099-10111 (2014).

77. Yoon, J. H. et al. LincRNA-p21 suppresses target mRNA translation. Mol. Cell. 47, 648-655 (2012).

78. Yang, Y. et al. TRMP, a p53-inducible long noncoding RNA, regulates G1/S cell cycle progression by modulating IRES-dependent p27 translation. Cell Death Dis. 9, 886 (2018).

79. Cho, S., Kim, J. H., Back, S. H. \& Jang, S. K. Polypyrimidine tract-binding protein enhances the internal ribosomal entry site-dependent translation of p27Kip1 mRNA and modulates transition from G1 to S phase. Mol. Cell Biol. 25, 1283-1297 (2005). 
80. Han, M. et al. Exosome-mediated IncRNA AFAP1-AS1 promotes trastuzumab resistance through binding with AUF1 and activating ERBB2 translation. Mol. Cancer 19, 26 (2020).

81. Gu, P. et al. A novel AR translational regulator IncRNA LBCS inhibits castration resistance of prostate cancer. Mol. Cancer 18, 109 (2019).

82. Wang, X. et al. A novel LncRNA HITT forms a regulatory loop with HIF-1alpha to modulate angiogenesis and tumor growth. Cell Death Differ. 27, 1431-1446 (2019).

83. Xu, T. P. et al. KLF5 and MYC modulated LINC00346 contributes to gastric cancer progression through acting as a competing endogeous RNA and indicates poor outcome. Cell Death Differ. 26, 2179-2193 (2019).

84. Lian, Y. et al. A novel IncRNA, LINC00460, affects cell proliferation and apoptosis by regulating KLF2 and CUL4A expression in colorectal cancer. Mol. Ther. Nucleic Acids 12, 684-697 (2018).

85. Sun, M. et al. LncRNA HOXA11-AS promotes proliferation and invasion of gastric cancer by scaffolding the chromatin modification factors PRC2, LSD1, and DNMT1. Cancer Res. 76, 6299-6310 (2016).

86. Pan, Y. et al. LncRNA H19 overexpression induces bortezomib resistance in multiple myeloma by targeting MCL-1 via miR-29b-3p. Cell Death Dis. 10, 106 (2019).

87. Santer, L., Bar, C. \& Thum, T. Circular RNAs: a novel class of functional RNA molecules with a therapeutic perspective. Mol. Ther. 27, 1350-1363 (2019).

88. Lei, M. et al. Translation and functional roles of circular RNAs in human cancer. Mol. Cancer 19, 30 (2020).

89. Liang, W. C. et al. Translation of the circular RNA circbeta-catenin promotes liver cancer cell growth through activation of the Wnt pathway. Genome Biol. 20, 84 (2019).

90. Yang, Y. et al. Novel role of FBXW7 circular RNA in repressing glioma tumorigenesis. J. Natl Cancer Inst. 110, 304-315 (2018).

91. Zhang, M. et al. A novel protein encoded by the circular form of the SHPRH gene suppresses glioma tumorigenesis. Oncogene 37, 1805-1814 (2018).

92. $\mathrm{Wu}, \mathrm{N}$. et al. Translation of yes-associated protein (YAP) was antagonized by its circular RNA via suppressing the assembly of the translation initiation machinery. Cell Death Differ. 26, 2758-2773 (2019).

93. Ng, W. L., Mohd Mohidin, T. B. \& Shukla, K. Functional role of circular RNAs in cancer development and progression. RNA Biol. 15, 995-1005 (2018).

94. Abdelmohsen, $\mathrm{K}$. et al. Identification of HuR target circular RNAs uncovers suppression of PABPN1 translation by CircPABPN1. RNA Biol. 14, 361-369 (2017).

95. Kim, J. et al. LncRNA OIP5-AS1/cyrano sponges RNA-binding protein HuR. Nucleic Acids Res. 44, 2378-2392 (2016).

96. Liu, Y. et al. Circular RNAMTO1 suppresses breast cancer cell viability and reverses monastrol resistance through regulating the TRAF4/Eg5 axis. Int. J. Oncol. 53, 1752-1762 (2018).

97. Chen, L. et al. Circ-MALAT1 functions as both an mRNA translation brake and a microrna sponge to promote self-renewal of hepatocellular cancer stem cells. Adv. Sci. (Weinh.) 7, 1900949 (2020).

98. Mao, Y., Zhang, L. \& Li, Y. circEIF4G2 modulates the malignant features of cervical cancer via the miR218/HOXA1 pathway. Mol. Med. Rep. 19, 3714-3722 (2019).

99. Zhang, $C$. et al. Hypoxia induces the breast cancer stem cell phenotype by HIFdependent and ALKBH5-mediated m(6)A-demethylation of NANOG mRNA. Proc. Natl Acad. Sci. USA 113, E2047-E2056 (2016).

100. Roundtree, I. A., Evans, M. E., Pan, T. \& He, C. Dynamic RNA modifications in gene expression regulation. Cell 169, 1187-1200 (2017).

101. Wen, J. et al. Zc3h13 regulates nuclear RNA m(6)A methylation and mouse embryonic stem cell self-renewal. Mol. Cell. 69, 1028-1038 (2018).

102. Yang, Y., Hsu, P. J., Chen, Y. S. \& Yang, Y. G. Dynamic transcriptomic m(6)A decoration: writers, erasers, readers and functions in RNA metabolism. Cell Res. 28, 616-624 (2018).

103. Wang, $X$. et al. N(6)-methyladenosine modulates messenger RNA translation efficiency. Cell 161, 1388-1399 (2015).

104. Zhou, J. et al. N(6)-methyladenosine guides mRNA alternative translation during integrated stress response. Mol. Cell. 69, 636-647 (2018).

105. Lin, $\mathrm{S}$. et al. The $\mathrm{m}(6) \mathrm{A}$ methyltransferase METTL3 promotes translation in human cancer cells. Mol. Cell. 62, 335-345 (2016)

106. Weng, $\mathrm{H}$. et al. METTL14 inhibits hematopoietic stem/progenitor differentiation and promotes leukemogenesis via mRNA m(6)A modification. Cell. Stem Cell. 22, 191-205 (2018).

107. Zhang, S. et al. m(6)A demethylase ALKBH5 maintains tumorigenicity of glioblastoma stem-like cells by sustaining FOXM1 expression and cell proliferation program. Cancer Cell. 31, 591-606 (2017).
108. Meyer, K. D. et al. 5'UTR m(6)A promotes cap-independent translation. Cell 163, 999-1010 (2015).

109. Zhou, J. et al. Dynamic m(6)A mRNA methylation directs translational control of heat shock response. Nature 526, 591-594 (2015).

110. Liu, T. et al. The m6A reader YTHDF1 promotes ovarian cancer progression via augmenting EIF3C translation. Nucleic Acids Res. 48, 3816-3831 (2020).

111. Shi, H. et al. YTHDF3 facilitates translation and decay of $N(6)$-methyladenosinemodified RNA. Cell Res. 27, 315-328 (2017).

112. Li, A. et al. Cytoplasmic m(6)A reader YTHDF3 promotes mRNA translation. Cell Res. 27, 444-447 (2017).

113. Ruzzenente, B. et al. LRPPRC is necessary for polyadenylation and coordination of translation of mitochondrial mRNAs. EMBO J. 31, 443-456 (2012).

114. Chen, E. et al. Fragile $X$ mental retardation protein regulates translation by binding directly to the ribosome. Mol. Cell. 54, 407-417 (2014).

115. Edupuganti, R. R. et al. N(6)-methyladenosine $(\mathrm{m}(6) \mathrm{A})$ recruits and repels proteins to regulate mRNA homeostasis. Nat. Struct. Mol. Biol. 24, 870-878 (2017).

116. Choi, J. et al. N(6)-methyladenosine in mRNA disrupts tRNA selection and translation-elongation dynamics. Nat. Struct. Mol. Biol. 23, 110-115 (2016).

117. Zhou, S. et al. FTO regulates the chemo-radiotherapy resistance of cervical squamous cell carcinoma (CSCC) by targeting beta-catenin through mRNA demethylation. Mol. Carcinog. 57, 590-597 (2018).

118. Niu, Y. et al. RNA N6-methyladenosine demethylase FTO promotes breast tumor progression through inhibiting BNIP3. Mol. Cancer 18, 46 (2019).

119. Hanson, G. \& Coller, J. Codon optimality, bias and usage in translation and mRNA decay. Nat. Rev. Mol. Cell Biol. 19, 20-30 (2018).

120. Sorensen, M. A. \& Pedersen, S. Absolute in vivo translation rates of individual codons in Escherichia coli. The two glutamic acid codons GAA and GAG are translated with a threefold difference in rate. J. Mol. Biol. 2222, 265-280 (1991).

121. Schmitt, B. M. et al. High-resolution mapping of transcriptional dynamics across tissue development reveals a stable mRNA-tRNA interface. Genome Res. 24, 1797-1807 (2014).

122. Pavon-Eternod, M. et al. tRNA over-expression in breast cancer and functional consequences. Nucleic Acids Res. 37, 7268-7280 (2009).

123. Goodarzi, H. et al. Modulated expression of specific tRNAs drives gene expres sion and cancer progression. Cell 165, 1416-1427 (2016).

124. Gingold, $H$. et al. A dual program for translation regulation in cellular proliferation and differentiation. Cell 158, 1281-1292 (2014).

125. Katz, F. N. \& Lodish, H. F. Transmembrane biogenesis of the vesicular stomatitis virus glycoprotein. J. Cell Biol. 80, 416-426 (1979).

126. Eichelbaum, K. et al. Selective enrichment of newly synthesized proteins for quantitative secretome analysis. Nat. Biotechnol. 30, 984-990 (2012).

127. Liu, J., Xu, Y., Stoleru, D. \& Salic, A. Imaging protein synthesis in cells and tissues with an alkyne analog of puromycin. Proc. Natl Acad. Sci. USA 109, 413-418 (2012).

128. Su, K. H. et al. HSF1 critically attunes proteotoxic stress sensing by mTORC1 to combat stress and promote growth. Nat. Cell Biol. 18, 527-539 (2016).

129. Chasse, H. et al. Analysis of translation using polysome profiling. Nucleic Acids Res. 45, e15 (2017).

130. Santos, D. A., Shi, L., Tu, B. P. \& Weissman, J. S. Cycloheximide can distort measurements of mRNA levels and translation efficiency. Nucleic Acids Res. 47, 4974-4985 (2019).

131. Murat, P. et al. RNA G-quadruplexes at upstream open reading frames cause DHX36- and DHX9-dependent translation of human mRNAs. Genome Biol. 19, 229 (2018).

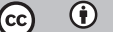

Open Access This article is licensed under a Creative Commons Attribution 4.0 International License, which permits use, sharing, adaptation, distribution and reproduction in any medium or format, as long as you give appropriate credit to the original author(s) and the source, provide a link to the Creative Commons license, and indicate if changes were made. The images or other third party material in this article are included in the article's Creative Commons license, unless indicated otherwise in a credit line to the material. If material is not included in the article's Creative Commons license and your intended use is not permitted by statutory regulation or exceeds the permitted use, you will need to obtain permission directly from the copyright holder. To view a copy of this license, visit http://creativecommons.org/licenses/by/4.0/.

(c) The Author(s) 2021 